\title{
Allocating resources in psychiatric hospitals according to casemix
}

\author{
Paul McCrone
}

There are advantages to be gained from a knowledge of the patient casemix that psychiatric hospltals, and community services, are likely to encounter. In particular the efficient allocation of resources would be better achieved if individuals could be 'placed' into groups. These groups would need to be clinically distinct and contain similar members. Previous methods of delining casemix have focused upon clinical diagnosis. However, the effectiveness of diagnosis in predicting resounce utilisation is limitied. A range of other factors could be used, including comorbidty, severty of illness, and previous hosplial contact.

There exists in the human psyche an apparent tendency to put people into boxes. Often the motives are undesirable, resulting from sexism, racism and the like. However, there may on occasion be justifiable and worthy reasons for wishing to group people together into categories. One such possible case is health care, where there are clear advantages to be gained from knowing what level of health care services a given patient requires and what the costs of these services are. This is especially relevant during the present era of change in health care provision. However, the extent to which groups may be formulated is likely to be limited. Some patients may naturally be grouped together; however, with others this may not be the case individuals with mental disorders being the most obvious example.

The recent reforms have placed great emphasis on the need for parsimony and quality of services, and the 1990 NHS and Community Care Act has particular implications for psychiatry. Gaining knowledge about patients has never been more important. The purchaser/provider split that it is at present evolving has resulted in the requirement for district health authorities to assess the needs of their populations in order to gauge what services are required and consequently what level of funding should be sought. Likewise GP fund-holders (who have been able to purchase non-in-patient mental health services from April of this year) and self-governing trusts would clearly benefit from a good reliable estimate of the service input a patient needs without this knowledge there may arise dis- incentives to treat patients where the level and costs of service provision are not known. If groups of 'similar' patients who required 'similar' levels of service input at a 'similar' cost were formulated then a major aid to resourcing health care would have been achieved. If this achievement were to be a reality, groups would need to be clearly distinct, contain relatively homogenous patients, and would need to be administratively manageable in number.

Prior to the NHS reforms there was little incentive in the UK to develop such groupings. However, there are likely to be future moves towards some form of prospective payment system for health care in the UK and, if groupings are to form the basis of such a system, alternative methods must be investigated and examined. Therefore, we have to draw on the experiences where this has been on the agenda, notably in the United States and its system of diagnosisrelated groups (DRGs).

DRGs group patients according to their principal diagnosis. For each DRG there is a related average length of hospital stay and hence an average cost. The number of DRGs is over 470; however, only 15 DRGs apply to mental disorders. Just from this information the three main problems with psychiatric DRGs should be apparent. First, they relate to in-patient stays. With the current drive towards community care. both in the United States and the UK, this makes them redundant for patients treated mainly or exclusively in the community. Second, the limited number of psychiatric DRGs means that they must be extremely broad-based. Indeed, one DRG covers all patients with a psychotic condition. Therefore, the predictive capabilities of the psychiatric DRGs is limited. Third, the basis for allocating patients to groups is the clinical diagnosis. This is not considered to be an effective indicator of the level, and range, of service input that a patient needs, even if diagnosis is more clearly defined.

Not surprisingly, psychiatric DRGs have not been a success in the United States, and the vast majority of psychiatric hospitals and psychiatric units are exempted from using them as a form of prospective payment. Better results have been 
attained with medical and surgical DRGs. However, this has still not removed the desire to create groupings for mental health care, in the UK and elsewhere, although the difficulties are clearly recognised.

What then are the other possibilities that exist for grouping psychiatric patients? A number of modifications have been made to DRGs. Refinements have included using comorbidity, patient characteristics, hospital setting, level of functioning, severity of illness, social setting, method of payment and regional characteristics. These have served to improve the predictive ability of DRGs, but not to any great extent. This is perhaps due to the fact that clinical diagnosis has still remained the cornerstone upon which modifications have been built. In addition, various alternative systems have been developed, such as disease staging, and groups have been created where diagnosis is still the determining factor, but where it is more specifically defined. This has not proved particularly fruitful. Also, measures of resource use other than length of in-patient stay have been used, such as number of nurse and other staff hours required by patients.

In the light of the problems with DRGs and related systems for psychiatry, what is the way forward in the UK? Healthcare resource groups (HRGs) have already been developed for general medical care but do not as yet cover psychiatry. This is as well, as they are strikingly similarly to DRGs. If acceptable groups are to be created for psychiatry, three important points should be considered. First, while the length of an inpatient stay may be a fairly robust measure of one area of resource use, it is irrelevant for some $80 \%$ of people with mental health problems, i.e. those who receive treatment primarily in the community. Instead, a measure of utilisation of community services is required. Second, diagnosis cannot credibly be the primary factor that determines how patients should be grouped. Other factors relating more clearly to the needs of patients are likely to be instrumental in determining what services should be purchased and provided. Diagnosis has a role, but in a hierarchy of grouping factors there is little justification for it to be given greatest weight. Third, the idea that the number of groups is going relatively low is overly optimistic. DRGs numbering 15 were wholly inadequate and alternative classification systems, with up to $\mathbf{7 0}$ or $\mathbf{8 0}$ groups, have not fared all that much better. The number of groups that would be required for an effective system that included all determining factors would be vast. However, given computer technology this would be feasible.

It may be that given the above difficulties a robust method of grouping psychiatric patients is not achievable. With this situation in mind, perhaps alternative approaches should be sought. One solution would be to have a variety of measures of resource use, relating to different aspects of community provision. In effect DRGs and related systems attach a single value to patients. A more realistic, and workable, method would ebb to allocate a range of values relating to a range of service inputs. The achievement of such a system if clearly a major challenge.

Paul McCrone, Health Economist, PRISM (Psychitric Research in Service Measurement). Instttute of Psychiatry, De Crespigny Park, London SE5 8AZ 